\title{
Web-based Surveys as an Alternative to Traditional Mail Methods
}

\author{
Christopher M. Fleming ${ }^{*}$ and Mark Bowden \\ School of Economics, University of Queensland, Brisbane QLD 4072, Australia. \\ * Corresponding author: Address: School of Economics, University of Queensland, Brisbane QLD 4072, \\ Australia. Tel: +61 7335 84967. Fax: +61 73846 6954. E-mail addresses: c.fleming@uq.edu.au (C \\ Fleming); m.bowden@uq.edu.au (M Bowden).
}

\begin{abstract}
Environmental economists have long used surveys to gather information about people's preferences. A recent innovation in survey methodology has been the advent of web-based surveys. While the Internet appears to offer a promising alternative to conventional survey administration modes, concerns exist over potential sampling biases associated with web-based surveys and the effect these may have on valuation estimates. This paper compares results obtained from a travel cost questionnaire of visitors to Fraser Island, Australia, that was conducted using two alternate survey administration modes; conventional mail and web-based. It is found that response rates and the socio-demographic makeup of respondents to the two survey modes are not statistically different. Moreover, both modes yield similar consumer surplus estimates.
\end{abstract}

Key words: Web-surveys; Mail survey; Sample bias; Travel cost method; Fraser Island. 


\section{Introduction}

Environmental economists have long used surveys to gather information about people's preferences. This is particularly true in the field of non-market valuation, where techniques such as the travel cost method, contingent valuation and choice modelling invariably employ some form of survey instrument. Conventional survey administration modes include mail, in-person, telephone and central site. More recently, the use of e-mail and web-based surveys has emerged as another option. The choice of survey mode requires consideration of several issues and, to date, no single mode has been proven unambiguously superior to the others (Champ, 2003).

The primary purpose of this paper is to test the suitability of web-based surveys for conducting non-market valuation studies, compared against an intercept mail survey. Mail surveys have much in common with web-based surveys in areas such as degree of personal contact between the interviewer and interviewee, access of the interviewee to the questionnaire, and freedom of the interviewee regarding the timing and method of answering the questionnaire. Valid comparisons should, therefore, be able to be drawn between the two modes. Moreover, mail surveys are the least costly and most common, suggesting that it is the mode most likely to be replaced, or supplemented by, web-based surveys. To our knowledge this is the first study to compare web-based and mail survey administration modes in a non-market valuation context.

Despite an emerging body of work employing web-based surveys ${ }^{1}$ to our knowledge only two non-market valuation studies explicitly compare web-based with other conventional survey administration modes. In the first of these, Berrens et al. (2003) compare a telephone sample with three web-based samples of a contingent valuation survey seeking U.S. household's willingness-to-pay for Senate ratification of the Kyoto Protocol. More recently, Marta-Pedroso et al. (2007) compare an in-person with a web-based sample of a contingent valuation survey seeking Portuguese residents' willingness-to-pay for the preservation of the cereal steppe of Castro Verde, Southern Portugal. Both studies find some differences in the socio-demographic

\footnotetext{
${ }^{1}$ See for example, Iraguen and Ortuzar (2004), Johnstone and Markandya (2006), Li et al. (2004), Schwappach and Strasmann (2006) and Tsuge and Washida (2003).
} 
makeup of respondents to the alternate modes and find the web-based mode to yield lower response rates and lower willingness-to-pay estimates than the conventional mode. Nonetheless, both conclude that the use of web-based surveys in contingent valuation research shows considerable promise.

We find our web-based survey to yield a sample not significantly different than our mail survey in terms of gender, age, income, education and country of residence of respondents, and at a substantially lower cost. Moreover, less than a $10 \%$ difference is found between the consumer surplus estimates derived from the two modes. These findings suggest the Internet has potential to become a valuable tool for non-market valuation practitioners.

The paper proceeds as follows. In Section 2 we discuss advantages and disadvantages of web-based surveys. Section 3 describes the study area, survey design and data collection. Section 4 compares the survey modes in terms of response rates, socio-demographic make up of respondents and surveying costs. Section 5 presents the travel cost analysis. Section 6 concludes.

\section{Advantages and disadvantages of web-based versus mail survey modes}

There are several features of web-based surveying that makes it attractive to researchers, of which the most commonly cited is cost. The comparatively low cost of web-based surveys is advantageous in that it enables large sample sizes, thus providing an increased potential for subgroup analysis and decreased sampling variance. Another often cited advantage of web-based surveys is the speed and accuracy of data collection. Web-based surveys can be up and running in a matter of days, with immediate access to results. Responses from online questionnaires can be automatically inserted into spreadsheets, databases or statistical packages. This not only saves time and money, but reduces human error in data entry and coding. Further, data can be collected continuously, regardless of time of day and day of week, and without geographical limitation (Madge, 2006; Manfreda, 2001). 
More than any other survey mode, web-based surveying allows innovative questionnaires to be developed. Visual and audio stimuli can be incorporated, prompts can alert respondents if they skip or incorrectly answer questions, drop-down boxes can present respondents with a range of possible answers, pop-up windows can provide additional information, ${ }^{2}$ questions can be ordered randomly, skip patterns may be built for ease of navigation, even multi-lingual formats are possible.

Moreover, colours, innovative question displays, split screens, animation, sound tracks and other advanced design features may make web-based questionnaires more appealing for respondents. Finally, the growth in email, online banking and bills being paid on the Internet suggest that, at least for some, the Internet is a more convenient medium than more traditional means of communication.

The most commonly cited disadvantages of web-based surveys are sample frame and nonresponse bias. The former is the non-random exclusion of individuals from the sample frame: in most populations there remains an enduring social and spatial divide in access and use of the Internet which can induce sample biases to any online research. Non-response bias is the bias introduced when respondents within the sample frame have very different attitudes or demographic characteristics to those who do not respond. This bias increases when different levels of technical ability are present among potential respondents, and it becomes a particular problem when response rates are low.

A further potential disadvantage of web-based surveying is the fact that the researcher often has no way of discerning if there are several respondents at one computer address, or if one respondent is completing a questionnaire from a variety of computers; although, as noted by Marta-Pedroso et al (2007), the use of cookies and server log files can largely address these concerns.

\footnotetext{
${ }^{2}$ The ability to provide more information is not necessarily advantageous; there is a considerable body of literature on information effects in non-market valuation. Reviews can be found in Blomquist and Whitehead (1998) and Munro and Hanley (2001).
} 


\section{Study area, survey design and data collection}

\subsection{Study area}

Situated less than five kilometres off the Queensland coast, at the southern end of the Great Barrier Reef and some 250 kilometres north of Brisbane (see Figure 1); Fraser Island is the largest sand island in the world (166 283 hectares) and one of Australia’s iconic natural attractions. Inscribed under criteria (vii) and (ix) ${ }^{3}$ on the World Heritage List in 1992, the Island attracts in excess of 300000 visitors per year. This level of visitation poses some significant environmental threats to the long-term preservation of the Island, with visitors contributing to a range of environmental problems including erosion, litter disposal, human-wildlife interactions and contamination of the Island's freshwater resources.

\section{[INSERT FIGURE 1]}

A recent report commissioned by the Queensland Environmental Protection Agency highlights the fact that many sites on Fraser Island are being used well beyond their capacity, suggesting that intervention may be required to ensure the Island's long-term sustainability (EDAW, 2002). Further, the Fraser Island World Heritage Area Scientific Advisory Committee recently recommended that visitor management be a priority action in order to minimise resource impacts and optimise the quality of visitor experiences and visitor safety (Queensland Environmental Protection Agency, 2002). This has prompted the Queensland Parks and Wildlife Service to consider access restrictions to Fraser Island.

To gain an appreciation of the potential welfare effects of any move to restrict access to the Island, it is useful to estimate the consumer surplus associated with current visitation. While organised tours already face access restrictions, and in the short- to medium-term are unlikely to

\footnotetext{
${ }^{3}$ Criteria (vii): to contain superlative natural phenomena or areas of exceptional natural beauty and aesthetic importance. Criteria (ix): to be outstanding examples representing significant on-going ecological and biological processes in the evolution and development of terrestrial, fresh water, coastal and marine ecosystems and communities of plants and animals (UNESCO, 2007).
} 
be restricted further, independent visitors currently face no restrictions and are the group most likely to have some form of restriction imposed upon them in the future. ${ }^{4}$ This application, therefore, using the zonal travel cost method, attempts to estimate the current (unrestricted) consumer surplus attributable to visiting Fraser Island by this particular group of visitors. This surplus, or at least some portion of it, is that most likely to be affected by future management interventions.

\subsection{Survey design and data collection}

The web-based survey was constructed using Macromedia Dreamweaver MX for Windows. To ensure the validity of any comparison between the two modes, the web-based questionnaire was constructed to look as similar as possible to that distributed on site. ${ }^{5} \mathrm{~A}$ link to the web-site was then placed on both the School of Economics website, and a number of other Fraser Island tourism information websites, with the simple invitation 'Visited Fraser Island recently? Click here to fill in a survey on the future management of the Island'.

Following the method of Wilson and Tisdell (2004), for the mail survey administration mode, visitors to Fraser Island were given questionnaires with pre-paid self addressed envelopes attached. This was to give respondents the opportunity to carefully consider replies in their own time. A total of two sampling occasions during 2006 were undertaken, the first in April and the second in August. In April 800 questionnaires were distributed over a period of seven days, in August 560 questionnaires were distributed over a period of eight days; reflecting the fact that August is a much quieter time of year on the Island. In both cases, questionnaires were distributed at a range of locations, predominantly in the lower third of the Island.

\footnotetext{
${ }^{4}$ Personal Communication, Ralph Henderson, Director, Tourism and Visitor Management, Parks Division, Queensland Parks and Wildlife Service, 3 June 2004.

${ }^{5}$ A copy of the web-based survey can be found on www.uq.edu.au/economics/survey.
} 


\section{Comparison of survey modes}

\subsection{Response rates}

Web-based and mail surveys can easily be ignored so getting a reasonable response rate can be challenging. Out of the 1360 mail questionnaires distributed over both sampling periods a total of 463 were returned, of which 33 (7.1\%) were unusable due to key questions (often relating to respondent's income and education) being left unanswered. Thus the mail survey mode yielded a response rate of $31.6 \%$.

Calculating response rates for web-based surveys presents some difficulties, there is no way of knowing how many individuals might have seen the survey, or links to the survey, but declined to participate. One way to deal with this is to use a visitation counter that keeps track of the number of times that a site has been accessed. The response rate can then be estimated by dividing the number of returned surveys by the visitation count. However, this is sure to underestimate response rates as the same individual may visit a site a number of times before submitting a completed questionnaire (Kay and Johnson, 1999). In this case, the survey site registered 442 'hits’, and 173 questionnaires were submitted, of which 27 (15.6\%) were unusable due to unanswered questions. Thus, while item non-response was higher, the webbased survey mode yielded a slightly higher overall response rate of $33.0 \%$ than the mail survey.

\subsection{Sample bias}

As seen in Table 1, very little difference is found between the gender, mean age and education of respondents to each survey mode, while respondents to the mail survey report a higher mean household income; a result consistent with that of Berrens et al. (2003). In contrast, Marta-Pedroso et al. (2007) found web-based survey respondents to be younger, more educated and have higher household incomes than respondents to their in-person survey. Also reported are chi-square $r X$ c Contingency Table test results. These suggest, at the $5 \%$ level of significance, that there are no observable differences between the two samples in terms of the 
distribution of gender $\left(\chi^{2}=0.0081<3.84=\chi_{1,0.05}^{2}\right)$, age $\left(\chi^{2}=0.6279<9.49=\chi_{4,0.05}^{2}\right)$, income $\left(\chi^{2}=4.3298<18.31=\chi_{10,0.05}^{2}\right)$ and education $\left(\chi^{2}=2.3925<7.81=\chi_{3,0.05}^{2}\right)$.

\section{[INSERT TABLE 1]}

In regards to the respondent's place of residence, $82 \%$ of respondents to the mail survey were residents of Australia, compared with 73\% of web-based survey respondents. As seen in Table 2, however, chi-square tests suggest the distribution of respondent's country of residence between survey modes is not significantly different $\left(\chi^{2}=6.0892<7.81=\chi_{3,0.05}^{2}\right)$.

70\% of Australian resident mail survey respondents were from Queensland, compared to only $51 \%$ of respondents to the web-based survey. As reported in Table 2, chi-square tests suggest the distribution of Australian respondent's state of residence between survey modes is significantly different $\left(\chi^{2}=18.2354>7.81=\chi_{3,0.05}^{2}\right)$. As Internet use in Queensland is not significantly lower than in the other States (Australian Bureau of Statistics, 2006), it seems likely that this result is driven by respondent recruitment, that is, Queenslanders, being more familiar with Fraser Island and its surrounds, are possibly less likely to visit promotional websites of the type used to recruit respondents to this survey. The differences in State of residence notwithstanding, researcher concerns about sample bias associated with web-based surveys in this case appear unfounded (at least in comparison to the mail alternative).

\section{[INSERT TABLE 2]}

\subsection{Cost advantage of web-based over mail survey modes}

As noted in Section 2, one of the key potential advantages of web-based over other survey administration modes is reduced cost. Excluding researcher time, out-of-pocket expenses for the mail survey include vehicle hire and fuel $(\$ 1730.50),{ }^{6}$ accommodation $(\$ 445.46)$, pre-paid envelopes and postage (\$274.18), and printing (\$1 047.20); a total of \$3 497.34 or $\$ 8.13$ per usable response. The only out-of-pocket expense associated with the web-based survey is the

\footnotetext{
${ }^{6}$ All figures are in AU \$. At the time of writing 1 AU \$ $=0.8140$ US \$; 0.4130 UK£.
} 
cost of software (\$283.00 or $\$ 1.94$ per usable response). ${ }^{7}$ Including researcher time widens the cost-gap between the two survey administration modes, to distribute mail questionnaires researchers spent a total of 15 days on Fraser Island; to develop the web version of the survey took approximately 6 days.

\subsection{Discussion}

We find that the web-based survey yields a sample not significantly different than the mail survey in terms of gender, age, income, education and country of residence of respondents, and at a substantially lower cost. This is an important finding; particularly given mail surveys are comparable to web-based surveys in many aspects of survey methodology. Moreover, as webbased surveying offers researchers many advantages over more conventional survey modes, these findings suggest that non-market valuation practitioners should consider the use of this survey administration mode in the future.

However, the finding of a significant difference in State of residence of Australian respondents between the alternate modes suggests the issue of respondent recruitment to webbased non-market valuation questionnaires deserves further consideration. As neither Berrens et al. (2003) nor Marta-Pedroso et al. (2007) report on the geographic distribution of respondents, this raises a previously unexplored issue and provides an area of future potential research.

\section{Travel cost analysis}

\subsection{Background}

The travel cost method seeks to place a value on recreational sites by using consumption behaviour in related markets. In other words, this is a non-market procedure whereby a value for a recreation site is obtained by considering how much people are prepared to spend to reach the site. Specifically, the costs of consuming the recreational amenity of a particular site are used as a proxy for price; these costs can include travel costs, entry fees, on-site expenditures and outlay

\footnotetext{
${ }^{7}$ This is a sunk cost; the marginal out-of-pocket expense of obtaining additional responses to the webbased survey is zero.
} 
on capital equipment. The method assumes weak complementarity between the recreational site and consumption expenditure. This implies that when consumption expenditure falls to zero, the marginal utility of visitation is also zero, or alternately the recreational site will only be valued if consumption expenditure is positive (Hanley and Spash, 1993). The method has become widely accepted and is generally regarded as one of the success stories of non-market valuation (Smith, 1993).

There are essentially two types of travel cost models, the individual, where the dependent variable is the number of trips per year (or per season) by individual users of a recreation site, and the zonal, where the dependent variable is the number of trips taken to the site by the population of a particular region or zone. While the former is more appropriate for local, frequently visited, sites, the latter is more appropriate for sites visited infrequently by travellers from afar, and is thus the model employed here.

There are two approaches that may be taken when estimating a zonal travel cost model, the traditional Clawson-Knetsch two-stage methodology (Clawson and Knetsch, 1966) and the gravity model, often used in geography and transport studies to model commuting decisions. The methodology and theoretical underpinnings of these two models are extensively discussed elsewhere (see for example, Hanley and Spash (1993)) and will not be revisited here. It is sufficient to note that the gravity model 'short-cuts', and has been shown to produce the same consumer surplus estimates as, the more cumbersome Clawson-Knetsch method. In all, the gravity approach is more elegant and has become increasingly popular with practitioners in recent times and is subsequently the approach taken in this paper.

\subsection{First-stage estimation procedure}

The first step in employing the gravity model is to determine a travel demand function (known as a 'trip generating function') based on the cost of travel and any other variables that may contribute to the explanation of visitation rates. The second step involves integrating this function for each zone using the respective zonal choke price (i.e. that price at which visitation falls to zero) as the upper limit for integration, and the current cost of travel from that zone as 
the lower limit. The integrals provide a measure of consumer surplus for each zone, which may then be summed to obtain the aggregate consumer surplus or recreational value of the site.

To obtain the trip generating function, zonal visitation rate was regressed against average zonal travel cost (TC) and three socio-demographic variables (age, income and education). Total travel costs are initially calculated per 'party' and include (where applicable): vehicle costs; air, bus and rail fares; recreational areas management vehicle access permit fees; and barge fees. Per-person travel costs are then estimated by dividing party travel costs by the number of adults in the party. ${ }^{8}$ As there is no theoretical reason for choosing one functional form over another, the linear, linear-log, log-linear, log-log and equations using the reciprocal of travel cost were all estimated.

Equations were assessed using AIC, SC, t-tests, adjusted $\mathrm{R}^{2}$ values, and consideration of autocorrelation and heteroskedasticity. The functional form of the preferred equations was found to be $\operatorname{Ln}(V R)=\alpha-\beta \cdot \operatorname{Ln}(T C)$ for both mail and web-based samples, as well as for a pooled data set. It was also found that the value of coefficients were similar in all three cases. As is typical with zonal travel cost studies, none of the socio-demographic variables were found to be significant, even at the $10 \%$ level. The results are outlined in Table 3.

\section{[INSERT TABLE 3]}

Substituting in current average zonal travel cost and multiplying by zonal population yields the estimated number of annual visits. Further, if these levels of visitation are divided by the annual number of vehicle permits issued to Australian residents, the estimated average number of adults per vehicle is obtained. As shown in Table 4, the estimated number of annual visits is 130909 for the mail, 131953 for the web-based and 138500 for the pooled sample. Given there are in excess of 300000 visitors annually, and many of these are on organized tours and thus excluded from the analysis, these figures seem reasonable. The estimated average number of

\footnotetext{
${ }^{8}$ It is assumed children do not contribute to paying costs and therefore represent an additional cost burden to the adults in the party.
} 
adults per vehicle is also reasonable when considering that the average number of adults per vehicle in the samples ranged from 3.4 to 3.6.

\section{[INSERT TABLE 4]}

\subsection{Consumer surplus estimation}

With the assumption that people will respond to increases in price in a similar way to increases in travel cost, the visitation levels corresponding to a schedule of travel costs was derived. In theory this involves incrementing the travel cost variable until zero visitation is obtained, thus identifying the choke price for each zone. However, due to the nature of a logarithmic function, it is not possible to obtain a zero level of visitation. Instead choke prices were identified at that point where the level of visitation in each zone reached one. For each sample, the zonal level of visitation $V_{i}$ was calculated using $V_{i}=V R_{i} \cdot N_{i}$, where $V R_{i}$ is the weighted average visitation rate. This rate is calculated as $V R_{i}=e^{\alpha-\beta \cdot \operatorname{Ln}\left(T C_{i}\right)}$, where the coefficients $\alpha$ and $\beta$ are as presented in Table $3, T C_{i}$ is the average current cost of travel from, and $N_{i}$ is the adult population of, zone $i$.

The trip generating functions were then integrated for each zone between the actual travel cost and the zonal choke price travel cost. Consumer surplus estimates for each zone are thus given by $C S_{i}=\int_{T C_{i}}^{T C_{i}+\Delta C_{\max }} V_{i} d T C$.

In order to account for multiple-site visitors, each zonal estimate of consumer surplus was adjusted according to the average zonal proportion of time spent on Fraser Island, relative to the time spent away from home on the trip; a method first suggested by Clough and Meister (1991). Results for all of the samples are summarised in Table 5.

\section{[INSERT TABLE 5]}




\subsection{Discussion}

The comparatively narrow range of the consumer surplus estimates reported in Table 5 go some way to confirming the findings of Section 4, namely that the alternate survey administration modes yield samples that are, for the most part, not significantly different. The relative magnitude of the estimates themselves demonstrates that the existing (unrestricted) recreational value of Fraser Island for independent visitors is large, in the region of \$180 million per annum or $\$ 1400$ per-person per-visit. This value, or at least some proportion of it, is potentially at risk if managers seek to restrict access to this group of visitors in the future and caution, therefore, is needed before moving to impose such a policy. That is not to suggest the imposition of access restrictions is necessarily a poor policy per se, simply that this potential loss of welfare needs to be balanced against any potential gains in value (including in non-use value) resulting from reduced visitor numbers. It is hoped that these estimates prove useful in supporting the evaluation of alternative future management options.

\section{Conclusion}

This paper tests the suitability of web-based surveys for conducting non-market valuation studies, compared against an intercept mail survey. It is demonstrated that the Internet has potential to become a valuable tool for non-market valuation practitioners, either in replacing, or supplementing, the more conventional mail survey administration mode. It is found that response rates and socio-demographic makeup of respondents to the alternate survey modes are not statistically different. Moreover, both modes yield similar consumer surplus estimates. While previous authors have suggested that web-based surveys perform adequately for contingent valuation studies, this research suggests they may also be used when employing a revealed preference non-market valuation technique such as the travel cost method.

In all, there is now a growing body of evidence that the web-based survey administration mode has considerable promise. Given its many advantages over more conventional survey modes, we suggest strong consideration be given to employing web-based surveys in nonmarket valuation applications in the future. 


\section{Acknowledgements}

This research was funded by a Queensland Department of Premier and Cabinet 'Smart State’ Grant. The authors would like to thank Craig Mosely for help with developing the webbased survey as well as Clem Tisdell, Averil Cook and participants at the $51^{\text {st }}$ Annual Conference of the Australian Agricultural and Resource Economics Society for their useful feedback on an earlier draft. Thanks also to the staff at the Queensland Parks and Wildlife Service for providing data and advice. Views expressed in this paper do not necessarily reflect those of Queensland Parks and Wildlife Service staff. All errors and omissions remain our own. 


\section{References}

Australian Bureau of Statistics, 2006. Household Use of Information Technology: 2005-06. Catalogue No. 8146.0, Canberra.

Berrens, R., Bohara, A., Jenkins-Smith, H., Silva, C., Weimer, D., 2003. The advent of Internet surveys for political research: A comparison of telephone and Internet samples. Political Analysis 11, 1-22.

Blomquist, G., Whitehead, J., 1998. Resource quality information and validity of willingness to pay in contingent valuation. Resource and Energy Economics 20, 179-196.

Champ, P., 2003. Collecting survey data for nonmarket valuation. In: Champ, P., Boyle, K., Brown, T. (Editors), A Primer on Nonmarket Valuation. Kluwer Academic Publishers, Dordrecht, pp.59-98.

Clawson, M., Knetsch, J., 1966. Economics of Outdoor Recreation, John Hopkins University Press, Baltimore.

Clough, P., Meister, A., 1991. Allowing for multiple-site visitors in travel cost analysis. Journal of Environmental Management 32, 115-125.

EDAW, 2002. Fraser Island Desired Site Capacities Study: Final Report. Queensland Environmental Protection Agency, Brisbane.

Hadwen, W., Arthington, A., 2003. The significance and management implications of perched dune lakes as swimming and recreation sites on Fraser Island, Australia. Journal of Tourism Studies 14, 35-44.

Hanley, N., Spash, C., 1993. Cost-Benefit Analysis and the Environment, Edward Elgar, Aldershot.

Iraguen, P., Ortuzar, J., 2004. Willingness-to-pay for reducing fatal accident risk in urban areas: An Internet-based web page stated preference survey. Accident Analysis and Prevention 36, 513-524. 
Johnstone, C., Markandya, A., 2006. Valuing river characteristics using combined site choice and participation travel cost models. Journal of Environmental Management 80, 237-247.

Kay, B., Johnson, T., 1999. Research methodology: Taming the cyber frontier. Techniques for improving online surveys. Social Science Computer Review 17, 323-337.

Li, H., Berrens, R., Bohara, A., Jenkins-Smith, H., Silva, C., Weimer, D., 2004. Would developing country commitments affect US households' support for a modified Kyoto Protocol? Ecological Economics 48, 329-343.

Madge, C., 2006. Online questionnaires: Advantages and disadvantages of online questionnaires. [Online]. Available: http://www.geog.le.ac.uk/ORM/questionnaires/quesads.htm. Accessed 1 November.

Manfreda, K. 2001. Web Survey Errors. PhD Thesis. University of Ljubljani.

Marta-Pedroso, C., Freitas, H., Domingos, T., 2007. Testing for the survey mode effect on contingent valuation data quality: A case study of web based versus in-person interviews. Ecological Economics (in press).

Munro, A., Hanley, N., 2001. Information, uncertainty, and contingent valuation. In: Bateman, I., Willis, K. (Eds.), Valuing Environmental Preferences: Theory and Practice of the Contingent Valuation Method in the US, EU, and Developing Countries. Oxford University Press, Oxford, pp.258-274.

Queensland Environmental Protection Agency, 2002. Australian National Periodic Report: Section II, Report on the State of Conservation of Fraser Island. Brisbane.

Schwappach, D., Strasmann, T., 2006. "Quick and dirty numbers"? The reliability of a statedpreference technique for the measurement of preferences for resource allocation. Journal of Health Economics 25, 432-448.

Smith, V. K., 1993. Nonmarket valuation of environmental resources: An interpretive appraisal. Land Economics 69, 1-26. 
Tsuge, T., Washida, T., 2003. Economic valuation of the Seto Inland Sea by using an Internet CV survey. Marine Pollution Bulletin 47, 230-236.

UNESCO, 2007. Fraser Island - UNESCO World Heritage Centre. [Online]. Available: http://whc.unesco.org/en/list/630. Accessed 8 March.

Wilson, C., Tisdell, C., 2004. Attitudes to entry fees to National Parks: Results and policy implications from a Queensland case study. Economic Analysis and Policy 34, 79-102. 
Figure 1: Location of Fraser Island, featuring main townships, lakes and unsealed roads.

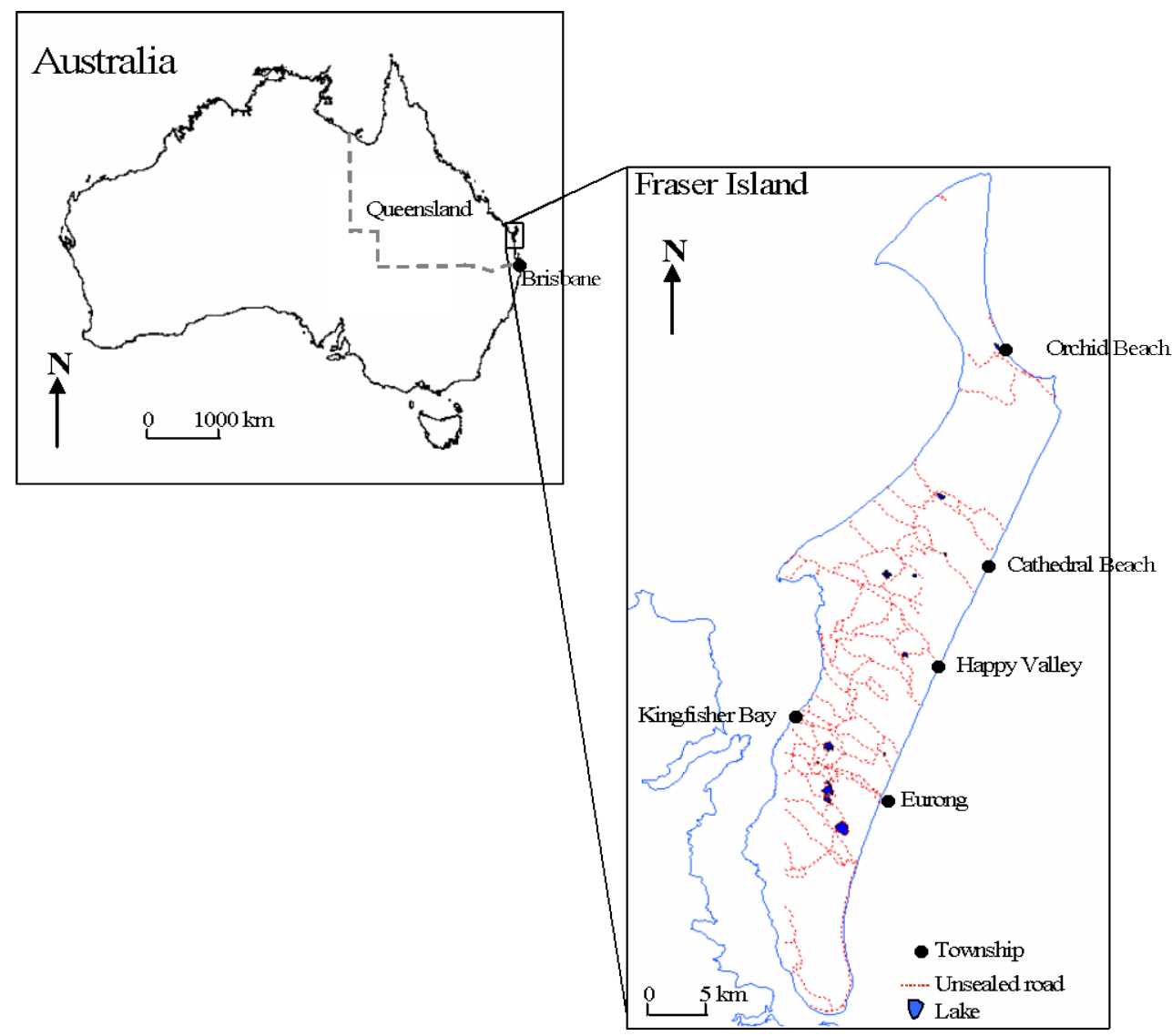

Source: Hadwen and Arthington (2003) 
Table 1: Gender, mean age, income and education of respondents

\begin{tabular}{lrrrr}
\hline Variable & Mail & Internet & Chi-squared & df \\
\hline Gender (proportion of sample male) $^{\mathrm{a}}$ & 51.40 & 51.01 & 0.0081 & 1 \\
Mean age (years) $^{\mathrm{a}}$ & 40.91 & 40.79 & 0.6279 & 4 \\
Mean income (gross weekly household AU \$) $^{\mathrm{b}}$ & 1,524 & 1,441 & 4.3298 & 10 \\
Mean highest educational attainment $^{\mathrm{c}}$ & 3.23 & 3.28 & 2.3925 & 3 \\
\hline
\end{tabular}

a Categories used were: 0-19, 20-29, 30-39, 40-49, 50-59, 60-69, 70-79 \& 80+. Because of the small number of respondents $(<$ $5)$, the two lowest $(0-19,20-29)$ and the two highest $(60-69,70-79)$ categories were pooled for the Chi-square test. Mean response values were calculated from the mid-points of category values. No respondent selected the 80+ category.

b Categories used were: <\$200, \$200-399, \$400-599, \$600-799, \$800-999, \$1 000-1 199, \$1 200-1 399, \$1 400-1 599, \$1 6001 799, \$1 800-1 999 \& \$2 000+. Mean response values were calculated from the mid-points of category value. For those who selected the highest category (\$2 $000+$ ) a figure of $\$ 2500$ was used.

c Categories used were: completed less than year 12 (highest year of schooling) or equivalent (coded 1), completed year 12 or equivalent (coded 2), trade certificate or equivalent (coded 3) and Bachelor or higher degree or equivalent (coded 4). 
Table 2: Place of residence of respondents

\begin{tabular}{|c|c|c|}
\hline Variable & Chi-squared & df \\
\hline Country of residence $^{a}$ & 6.0892 & 3 \\
\hline State of residence (Australian residents only) ${ }^{b}$ & $18.2354 *$ & 3 \\
\hline \multicolumn{3}{|c|}{$\begin{array}{l}\text { a Categories used were: Australia, North America, UK \& Ireland, Europe, and 'other'. } \\
\text { Because of the small number of respondents }(<5) \text {, the categories of North America and } \\
\text { 'other' were pooled for the Chi-square test. }\end{array}$} \\
\hline \multicolumn{3}{|c|}{$\begin{array}{l}\text { Because of the small number of respondents }(<5) \text {, respondents from South Australia and } \\
\text { Western Australia were pooled for the Chi-square test. }\end{array}$} \\
\hline
\end{tabular}


Table 3: Preferred first-stage estimation equations

\begin{tabular}{lrrr}
\hline & Mail & Internet & Pooled \\
\hline Constant $(\alpha)$ & 11.2257 & 11.2464 & 11.3301 \\
& $(0.6537)$ & $(0.7707)$ & $(0.7352)$ \\
Independent variable $(\beta)$ & -1.0676 & -1.0697 & -1.0832 \\
& $(0.0998)$ & $(0.11191)$ & $(0.1123)$ \\
$\operatorname{adjR}^{2}$ & 0.825 & 0.809 & 0.802 \\
\hline
\end{tabular}

Note: Standard errors are provided in brackets. All parameters are significant to the $5 \%$ level. 
Table 4: Estimated level of visitation and adults per vehicle

\begin{tabular}{lrrr}
\hline & \multicolumn{1}{c}{ Mail } & Internet & \multicolumn{1}{c}{ Pooled } \\
\hline Level of visitation (adults) & 130909 & 131933 & 138500 \\
Adults per vehicle & 3.4 & 3.2 & 3.4 \\
\hline
\end{tabular}


Table 5: Summary of consumer surplus estimates (AU \$)

\begin{tabular}{lrrr}
\hline & \multicolumn{1}{c}{ Mail } & Internet & \multicolumn{1}{c}{ Pooled } \\
\hline Total per annum (000) & $\$ 191353$ & $\$ 178192$ & $\$ 183855$ \\
Per-person per-visit & $\$ 1461$ & $\$ 1351$ & $\$ 1327$ \\
\hline
\end{tabular}

